News

\section{Put cancer on a diet!}

\section{News}

Should doctors be prescribing weight loss as a treatment for breast cancer? A recent article in the New York Times talked about this by highlighting a study that is has gotten underway this summer. This study has been initiated by the Susan F. Smith Center for Women's Cancers at the Dana Farber Cancer Institute in Boston. The researchers have recruited 3200 women from the US and Canada with recently diagnosed Stage 2 or Stage 3 breast cancer. The women must be overweight with a BMI of at least 27 and they must have certain characteristics to their breast cancer. They have to be able to walk at least a couple of blocks and have a life expectancy of 5years. They will be randomly assigned to either a telephone-based weight loss program or to a control group as a comparison. The weight loss program is basically a walking program and telephone reminders to exercise, as well as suggestions on how to keep up with the program. Previous studies have shown that if patients receive telephone reminders, they are more likely to keep up with the exercise program. These patients will be followed for 10years to see if the exercise program prevents their cancer from coming back.

There are studies that are decades old that demonstrate that obese and overweight women are more likely to die of their breast cancer. Two years ago a meta-analysis was done of over 80 studies involving over 200,000 women; this study showed that women who were obese when diagnosed had a $41 \%$ greater risk of death, while women who were in the overweight category but had a BMI less than 30 had a $7 \%$ risk of dying from their cancer. Although this study is specific for breast cancer, it has been well-established in other cancers that obesity puts us in a higher risk category for cancer. This is true in colon cancer, for example. The changes in our bodies that happen with obesity include higher insulin and glucose levels, inflammation, and higher levels of certain proteins-all of which seem to give fuel to

\section{Oopen Access CrossMark}

Volume 2 Issue 4 - 2016

\author{
Maria Palafox \\ University of Texas Medical School San Antonio, USA
}

Correspondence: Maria Palafox, South Texas Breast Surgery, University of Texas Medical School San Antonio, USA, Tel 210219-8480,Email maria.palafox@aol.com

Received: August 22, 2016 | Published: August 29, 2016

cancer growth. All these things send a message to cancer a cell telling them it is a good time to grow and the nutrition that cancer needs is there in obese patients.

Weight loss is not easy. Consider this, however: there was a trial a few years back that also tested a telephone-based weight loss program and found that women who lost only 4 to $5 \%$ of their body weight lowered their risk of cancer. That is only 7-10pounds for a person who weighs about 200 pounds. So even though most of us would like to lose more than that, 7 to 10pounds is a number that we can hope to reach. Talk to your doctor about a walking program; it will not only help diabetes and blood pressure, but can also lower your risk for cancer by helping you lose weight. Put cancer on a diet!

\section{Acknowledgements}

None.

\section{Conflict of interest}

The author declares no conflict of interest. 